\title{
Whole-Body Vibration Promotes Beneficial Changes on the Anterior Tibial Muscle Histomorphometry of Hypothalamic Obese Rats
}

\author{
M. F. Zazula', C. Bergmann Kirsch², J. L. Theodoro², C. de Toni Boaro², D. F. Saraiva², \\ S. Gonçalves de Oliveira ${ }^{1}$, B. Zanardini de Andrade ${ }^{2}$, A. L. Peretti ${ }^{2}$, K. Naliwaiko', \\ G. R. Flor Bertolini ${ }^{2}$, R. M. Costa ${ }^{2}$, L. de Fátima Chasko Ribeiro ${ }^{2}$ \\ 1 Departament of Cellular Biology, Sector of Biological Sciences, Federal University of Paraná, Curitiba, Paraná, Brazil \\ 2 Center of Biological Sciences and of Health, Western Paraná State University, Cascavel, Paraná, Brazil
}

\section{CORRESPONDING AUTHOR:}

Matheus Felipe Zazula

Departamento de Biologia Celular

Setor de Ciências Biológicas

Universidade Federal do Paraná

Avenida Coronel Francisco

H. dos Santos 100

81530-000 Curitiba

Paraná, Brasil

E-mail: matheuszazula@gmail.com

DOI:

10.32098/mltj.04.2021.07

LEVEL OF EVIDENCE: 1B

\begin{abstract}
SUMMARY
Background. Due to the deleterious effects of obesity on muscle tissue and the search for tools to reverse these losses, it is important to understand the effect of physical exercises on the muscle structure of obese individuals. This study aimed to analyze the effect of wholebody vibration (WBV) on the histomorphological parameters of the anterior tibial muscle using the monosodium 1-glutamate (MSG) obesity model.

Methods. MSG-obese rats that were exposed to WBV on a vibrating platform with a frequency of $60 \mathrm{~Hz}$, the amplitude of $2 \mathrm{~mm}$, three times/week, $10 \mathrm{~min} /$ day, for eight weeks (from postnatal day (PN) 80 to PN136). The histomorphology of the anterior tibial muscle was evaluated.

Results. When performing a WBV exercise, the animals showed altered structural responses in the MSG animals, such as reduced muscle mass, increased connective tissue, and nuclear activity. The WBV reduced the extracellular matrix and the nuclear activity in the MSG animals, showing efficiency in the protocol.

Conclusions. Even with the aggressive character of the MSG model, the WBV exercise was able to induce repair to the muscle tissue of these animals, thus being a safe protocol for use in similar conditions.
\end{abstract}

KEY WORDS

Exercise therapy; extracellular matrix; monosodium glutamate; skeletal muscle; WholeBody Vibration.

\section{BACKGROUND}

According to the World Health Organization, obesity is a pandemic that affects approximately 650 million people (1), who are subject to several associated comorbidities. The accumulation of adipose tissue can negatively influence the process of muscle remodeling, promoting the reduction of muscle mass, potentiating the deleterious effects on the locomotor system (2). Overweight caused by obesity has several negative effects on the locomotor system, including joint problems, pain, and reduced locomotion (3).
This excess of adipose tissue is one of the main responsible for the metabolic changes of obesity (4), one of the main characteristics of which is the increase in the secretion of pro-inflammatory cytokines. The constant maintenance of high levels of these inflammatory molecules stimulates the reduction of synthesis and increase of protein degradation, consequently leading to a reduction in muscle mass and strength (5). With persistent muscle inflammation, satellite cells, responsible for the regeneration of muscle fibers, have their recruitment reduced and there is a greater proliferation of fibroblasts, and thus, there is an increase in 
intramuscular connective tissue that contributes to muscle changes and weakness (6).

To minimize part of the deleterious effects of obesity and its comorbidities, several experimental methods are employed, and physical exercise is effective and has few side effects. As they are low-invasive and low-cost modalities, they are easily adopted in the treatment of obesity leading to improved quality of life (7). The Whole-Body Vibration (WBV), obtained through vibrating platforms, is an alternative for people who, due to their physical conditions, do not adhere to traditional forms of physical exercise, and dependent on a low-impact activity (5).

WBV acts directly on the muscle, increasing the capacity for muscle contraction and relaxation (8), stimulating protein synthesis, accelerating regeneration, and improving vascularization (9). However, even knowing the effects of WBV on some muscle and bone parameters, the effects of this exercise modality associated with obesity models are not well understood. Thus, the present study aimed to evaluate the effects of WBV on the histomorphology of the anterior tibial muscle of Wistar rats with obesity induced by monosodium glutamate (MSG).

\section{METHODS}

\section{Animals and experimental model}

This study had an experimental character with a completely randomized design (DIC), with a level of evidence $1 \mathrm{~b}$. For the present study, 32 male Wistar rats were used, kept in standard polypropylene boxes, in an environment with a temperature of $22 \pm 1{ }^{\circ} \mathrm{C}$, with a photoperiod of 12 hours, with free access to water and feed. Initially, the animals were randomly separated into two experimental groups $(\mathrm{n}=16)$ : 1) CTL, whose animals received hyperosmotic saline, and 2) MSG, whose animals were induced to obesity with monosodium glutamate. Of these, each group was subdivided into two other experimental groups $(n=8)$ : a) CTL-SED control group, b) MSG-SED - obese group c) CTL-WBV - control group trained with WBV and d) MSG-WBV an obese group trained with WBV (5). All procedures were conducted according to the ethics parameters described in this journal (10).

In the very first days of life, with an initial body weight between six and seven grams and that comprised the MSG-SED and MSG-WBV groups received intradermal injections of monosodium glutamate (MSG) at a dose of 4 $\mathrm{mg} / \mathrm{g}$ of body weight during the first 5 days deity. For those composing the CTL-SED and CTL-WBV groups, during the same period, hyperosmotic saline solution $(12.5 \%)$ was administered at a dose of $1.25 \mathrm{mg} / \mathrm{g}$ of body weight.

\section{Whole-Body Vibration protocol}

The platform used was the professional tri-plane Vibro Oscillatory model of the Arktus brand, with adapted protocol a frequency of $60 \mathrm{~Hz}$, the amplitude of two millimeters, for 10 minutes $(5,7)$, from the animals' 80 days of age, three times a week, on alternate days, making a total of eight weeks of treatment (11). To place the animals on the platform, to contain them and carry out the training with several animals simultaneously, a white wooden MDF support was used, compartmented in eight stalls $13 \mathrm{~cm}$ wide, $19 \mathrm{~cm}$ long, and height of 25 centimeters. At each training, the animals were rotated between the bays to minimize bias due to different vibration frequencies in different areas of the platform (figure 1).
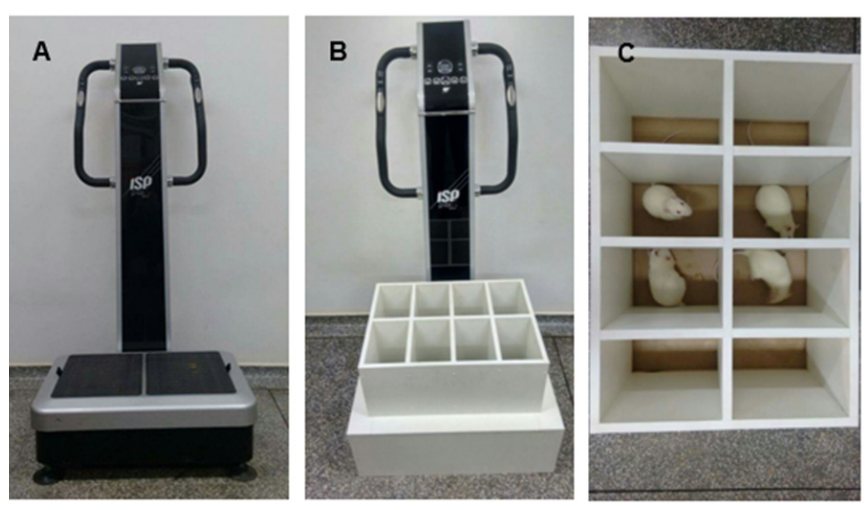

Figure 1. (A) Vibrating platform, (B) front view and (C) top view.

\section{Collection and preparation of the Tibialis Anterior Muscle}

At 142 days of age, the animals were weighed, desensitized in a $\mathrm{CO}_{2}$ chamber, and euthanized by guillotining. The retroperitoneal (RETRO) and perigonadal (PERIG) fats were removed, their weight measured and normalized to $100 \mathrm{~g}-1 \mathrm{~g}$ body weight, and the Lee Index $(\sqrt[3]{\text { bodyweight }}$ / nasal-anal length $\times 1000$ ) was calculated (7). The Tibialis Anterior muscle was dissected and fixed in metacarn for 24 hours, stored in 70\% alcohol until processing for inclusion in paraffin blocks, where they were first dehydrated in increasing alcoholic series and diaphanized in N-butyl alcohol. The blocks were cut transversely in a microtome to a thickness of five $\mu \mathrm{m}$ and stained in Hematoxylin and Eosin, and Masson's Trichromic $(5,12)$.

\section{Histomorphological analyzes}

The materials, stained with Hematoxylin and Eosin and Masson's Trichromic, were photomicrographed using a 
photomicroscope with an Olympus BX60 ${ }^{\circledR}$ coupled camera (Tokyo, Japan) with a 40x objective, totaling 12 images per animal in each technique. The morphological analyzes were performed in the Image ProPlus 6.0 program, and in each image the muscle fasciculus was scanned to randomly select ten fibers, thus totaling 120 fibers per animal (5).

From these fibers, the shape and organization and density (DENS) the positions of the nuclei, and the architecture of the connective tissue were analyzed, and the cross-sectional area (CSAF), larger (LDF), and smaller (SDF) diameters, was measured. The numbers of central (NC\%) and peripheral nuclei were also quantified, as well as the area of these nuclei (CSAN). The number of fibers and nuclei were used to calculate the nucleus to fiber ratio $(\mathrm{N} / \mathrm{F})$ and the area ratio of the nuclei to the sarcoplasm (AN/AF) area $(5,11)$. As for the analysis of connective tissue, the GIMP 2.0 program was used, and the quantification and comparison of the total number of pixels in the image with the number of connective tissue pixels were performed, to define the total connective tissue present in the visual field. Thus, this quantification considered the percentage of pixels referring to the total connective tissue (CON), epimysium (EPI), perimysium (PER), and endomysium (END) $(11,12)$.

\section{Statistical analysis}

All variables were analyzed by the normality (Shapiro-Wilk test) and homoscedasticity (Bartlet test) and those that were in agreement with such assumptions, were analyzed by Two-Way Analysis of Variance (ANOVA) follow by the posthoc Tukey-HSD test, to examine the interaction of obesity (MSG) and whole-body vibration (WBV). When the assumptions were not in agreement, the Kruskal-Wallis test was performed followed by the posthoc Dunn test. All analyses were performed with a level of significance $\alpha=0.05$.

Then, the matrices of the variables were standardized and analyzed using the principal component analysis (PCA). With the PCA, factorial loads are established for each variable and analyzed in response components. The data provided by the PCA is reduced, the data overlays are removed, and the most representative linear units of the data are known. The factor loads of the main components were evaluated in terms of statistical significance using a Two-Way Analysis of Variance (ANOVA) follow by the posthoc Tukey-HSD test (7). As the main components (PC) are ordered in decreasing order of importance for a structure of variance of the data set, the greater the retention of the total variance in a smaller number of linear formulas, the better the application of the procedure to the experimental data. All procedures were performed in software $\mathrm{R}$ version 4.0.3 (13).

\section{RESULTS}

\section{Body Parameters}

Bodyweight was affected only by MSG ( $p<0.00001)$, with no modification by the WBV factor. The Lee index showed a significant difference due to the fixed effects of MSG and WBV. An increase in means was observed in the MSG groups $(\mathrm{p}<0.0001)$ and a significant reduction in the WBV groups $(\mathrm{p}=0.01$; table I). Only the MSG resulted in an increase in weight of the retroperitoneal and perigonadal fats compared to the respective controls $(\mathrm{p}<0.0001$; $<<$ 0.0001 , respectively; table I). There was no significant interaction between the fixed factors, which means that exercise did not change the effect of MSG on these variables.

\section{Morphological analysis}

The CTL-SED (figure 2 A) animals have polygonal fibers, with the nuclei in a peripheral location, just below the sarcoplasmic membrane. The extracellular matrix (figure 2 E) organized in endomysium, intimately in contact with the sarcoplasmic membrane, individually covers each muscle fiber. The fibers are organized in muscle fascicles, delimited by the perimysium that allows the entry of blood vessels and nerves. Meanwhile, the animals of the MSG-SED (figure 2 B) present both polygonal fibers and fibers with a rounded shape and of smaller size, with a greater occurrence of central nuclei. It is also possible to observe a greater presence of connective tissue in all muscle wraps (figure $\mathbf{2} \mathbf{F}$ ). However, the CTL-WBV (figure 2 C) and OBS-WBV (figure 2 D) animals showed predominantly polygonal muscle fibers, with a smaller caliber than the respective sedentary groups, and less occurrence of central nuclei. In the case of connective wraps (figure $\mathbf{2} \mathbf{~ G , ~ F}$ ), it is also possible to notice less deposition throughout the extracellular matrix of these groups.

\section{Morphometrical analysis}

When assessing the CSAF, there was no interaction between the factors ( $p=0.457)$. However, MSG animals showed lower values when compared to CTL ( $\mathrm{p}<0.0001)$. Meanwhile, WBV animals showed a decrease in CSAF when compared to $\operatorname{SED}(\mathrm{p}=0.003)$. Likewise, there was no interaction between the factors in the LDF analysis $(\mathrm{p}=0.083)$. However, MSG animals showed lower values when compared to CTL ( $\mathrm{p}<$ 0.0001). Following the same pattern, WBV animals showed a reduction in LDF when compared to SED ( $\mathrm{p}<0.0001)$. When evaluating the SDF, there was an interaction between the factors $(\mathrm{p}<0.0001)$, with no difference between the CTL and MSG animals $(\mathrm{p}=0.992)$. However, CTL-WBV animals 
Table I. Average \pm Standard Deviation of the values of body and morphometric parameters of Wistar rats.

\begin{tabular}{|c|c|c|c|c|c|c|c|}
\hline \multicolumn{4}{|c|}{ GROUPS } & \multicolumn{4}{|c|}{ p VALUES } \\
\hline & CTL-SED & CTL-WBV & MSG-SED & MSG-WBV & MSG & WBV & INT \\
\hline BW & $407.33 \pm 12.85^{\mathrm{Aa}}$ & $400.00 \pm 17.49^{\mathrm{Aa}}$ & $317.60 \pm 9.03^{\mathrm{Ba}}$ & $307.78 \pm 11.51^{\mathrm{Ba}}$ & $<0.0001$ & 0.875 & 0.248 \\
\hline RETRO & $1.20 \pm 0.36^{\mathrm{Ba}}$ & $1.22 \pm 0.28^{\mathrm{Ba}}$ & $1.97 \pm 0.43^{\mathrm{Aa}}$ & $2.05 \pm 0.45^{\mathrm{Aa}}$ & $<0.0001$ & 0.728 & 0.817 \\
\hline PERIG & $1.31 \pm 0.14^{\mathrm{Ba}}$ & $1.16 \pm 0.36^{\mathrm{Ba}}$ & $1.70 \pm 0.40^{\mathrm{Aa}}$ & $1.85 \pm 0.41^{\mathrm{Aa}}$ & 0.0001 & 0.975 & 0.229 \\
\hline SDF & $43.80 \pm 2.07^{\mathrm{Aa}}$ & $36.24 \pm 1.95^{\mathrm{Bb}}$ & $40.571 \pm 1.70^{\mathrm{Ba}}$ & $39.46 \pm 1.92^{\mathrm{Aa}}$ & 0.992 & $<0.0001$ & $<0.0001$ \\
\hline DENS & $227.36 \pm 35.39^{\mathrm{Bb}}$ & $275.56 \pm 22.34^{\mathrm{Ba}}$ & $308.65 \pm 36.93^{\mathrm{Ab}}$ & $392.32 \pm 47.49^{\mathrm{Aa}}$ & $<0.0001$ & $<0.0001$ & 0.182 \\
\hline $\mathrm{N} / \mathrm{F}$ & $2.10 \pm 0.10^{\mathrm{Aa}}$ & $1.92 \pm 0.03^{\mathrm{Ab}}$ & $1.74 \pm 0.09^{\mathrm{Ba}}$ & $1.61 \pm 0.17^{\mathrm{Bb}}$ & $<0.0001$ & $<0.0001$ & 0.519 \\
\hline $\mathrm{NC} \%$ & $0.89 \pm 0.19^{\mathrm{Bb}}$ & $0.77 \pm 0.20^{\mathrm{Bb}}$ & $1.31 \pm 0.38^{\mathrm{Aa}}$ & $0.54 \pm 0.17^{\mathrm{Bb}}$ & 0.289 & $<0.0001$ & 0.001 \\
\hline EPI & $2.32 \pm 0.22^{\mathrm{Bb}}$ & $2.15 \pm 0.16^{\mathrm{Bb}}$ & $4.05 \pm 021^{\mathrm{Aa}}$ & $2.59 \pm 0.12^{\mathrm{Ab}}$ & $<0.0001$ & $<0.0001$ & $<0.0001$ \\
\hline PER & $1.08 \pm 0.14^{\mathrm{Bb}}$ & $1.22 \pm 0.08^{\mathrm{Bb}}$ & $2.37 \pm 0.16^{\mathrm{Aa}}$ & $1.56 \pm 0.14^{\mathrm{Ab}}$ & $<0.0001$ & $<0.0001$ & $<0.0001$ \\
\hline END & $1.15 \pm 0.07^{\mathrm{Ba}}$ & $0.89 \pm 0.23^{\mathrm{Bb}}$ & $1.82 \pm 0.23^{\mathrm{Aa}}$ & $1.12 \pm 0.09 \mathrm{Ab}$ & $<0.0001$ & $<0.0001$ & 0.001 \\
\hline
\end{tabular}

CTL-SED: sedentary control group; CTL-WBV: trained control group; MSG-SED: sedentary obese group, MSG-WBV: trained obese group. Capital and different letters represent isolated differences from the MSG factor; lowercase and different letters represent isolated differences from the WBV. BW: body weight (g); LEE: Lee index (g/ $\left.\mathrm{cm}^{3}\right)$; RETRO: retroperitoneal adipose tissue (g/100 g); PERIG: perigonadal adipose tissue (g/100 g); CSAF: cross-sectional area of the muscle fiber $\left(\mu \mathrm{m}^{2}\right)$; LDF: larger diameter of the muscle fiber $(\mu \mathrm{m})$; SDF: smaller diameter of the muscle fiber ( $\left.\mu \mathrm{m}\right)$; DENS: density of muscle fibers (fibers $/ \mathrm{mm}^{2}$ ); N/F: core to fiber ratio; NC\%: percentage of central nuclei; CSAN: cross-sectional area of the nucleus ( $\left.\mu \mathrm{m}^{2}\right)$; AN/AF: a ratio of the nucleus area to sarcoplasm area; CON: total connective tissue (\% pixels); EPI: connective tissue of the epimysium (\% pixels); PER: perimysium connective tissue (\% pixels); END: connective tissue of the endomysium (\% pixels).

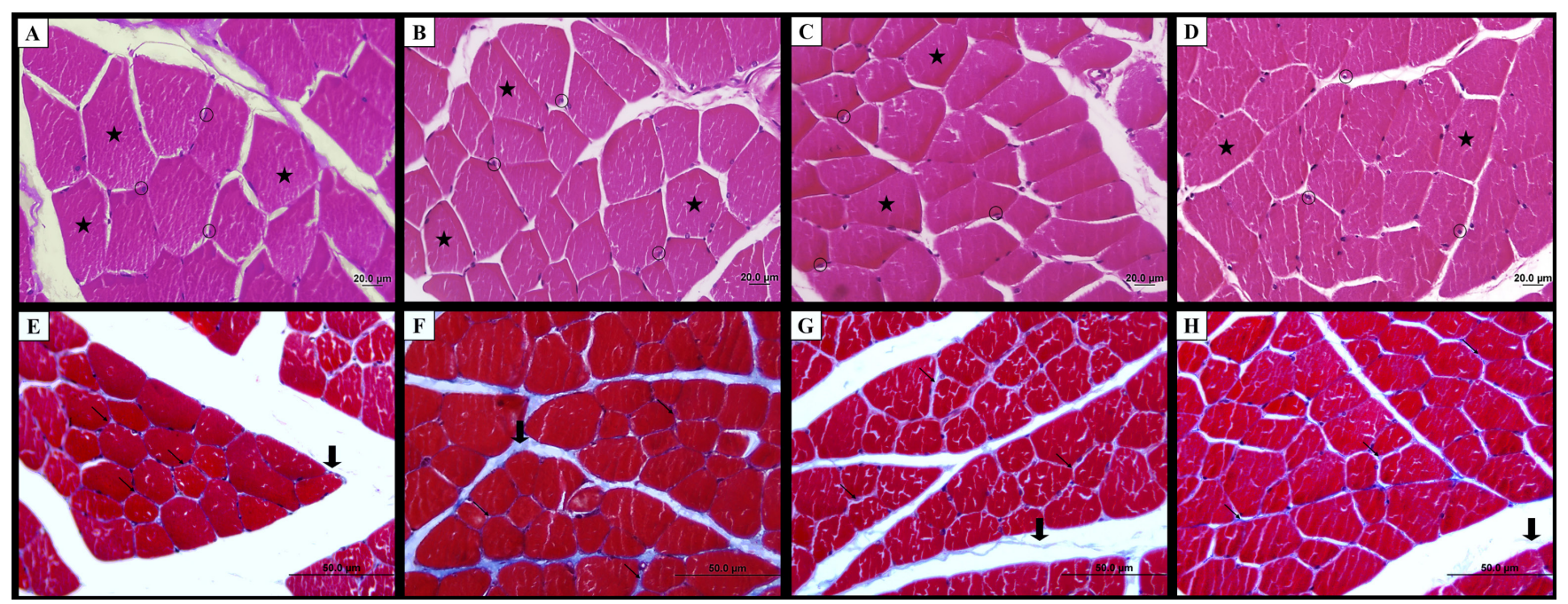

Figure 2. Photomicrographs of the anterior tibial muscle of Wistar rats at 142 days of age.

Cross-section, hematoxylin, and eosin staining (A-D), and Masson's trichrome (E-H). (A, E) Sedentary control group (CTL-SED); (B, F) Sedentary obese group (MSG-SED); (C, G) Trained control group (CTL-WBV); (D, H) Trained obese group (MSG-WBV). Polygonal-shaped fibers (stars); peripheral nuclei (circles). Connective tissue corresponding to perimysium (thick arrows) and endomysium (thin arrows). 
showed a reduction in SDF when compared to CTL-SED ( $p<0.0001)$. In the case of DENS, there was no interaction between the factors $(\mathrm{p}=0.182)$. However, there was an increase in MSG animals when compared to CTL ( $\mathrm{p}<$ 0.0001). Likewise, the WBV animals showed higher averages than the SED $(\mathrm{p}<0.0001)$ (table I).

When comparing the N/F values, there was no interaction between the factors $(p=0.519)$. However, there was a reduction of this ratio in MSG animals when compared to CTL $(\mathrm{p}<0.0001)$. Likewise, there was a reduction in $\mathrm{N} / \mathrm{F}$ in WBV animals compared to SED $(\mathrm{p}<0.0001)$. In the case of $\mathrm{NC} \%$, there was an interaction between the factors ( $\mathrm{p}=0.001)$, but there was no difference between the CTL and MSG animals $(\mathrm{p}=0.289)$. However, WBV reduced $\mathrm{NC} \%$ in MSG animals $(\mathrm{p}<0.0001)$. When comparing the CSAN values, there was no interaction between the factors ( $p=0.723$ ), and no difference between the CTL and MSG animals $(p=0.365)$. However, the WBV promoted an increase in CSAN in trained animals $(p=0.001)$. Similarly, there was no interaction between factors $(p=0.524)$ in AN/AF, and there was also no difference between CTL and MSG animals $(p=0.968)$. However, WBV promoted an increase in AN/AF when compared to SED animals ( $\mathrm{p}=$ 0.01) (table I).

When assessing connective tissue variables, there was an interaction between factors in the CON $(\mathrm{p}<0.0001)$, EPI $(\mathrm{p}<0.0001)$, PER $(\mathrm{p}<0.0001)$ and END $(\mathrm{p}=0.001)$. In all envelopes, MSG animals had higher connective tissue averages than CTL $(p<0.0001)$. Likewise, the exercise promoted a reduction in all connective wraps in MSG-WBV animals when compared to MSG-SED ( $\mathrm{p}<0.0001$ ). Only in END, the exercise promoted the same reduction in CTL-WBV animals when compared to CTL-SED ( $p<0.0001)$ (table I).

\section{Principal components analysis}

After creating the PCs, it was observed that only 3 dimensions correspond to $84.47 \%$ of data exploration. Therefore, these 3 components were used for the development of the analysis. The first dimension was called the Extracellular Matrix (CON, EPI, PER, END), the second was called Muscle Mass (CSAF, LDF, SDF, DENS, AN/AF, N/F), and the third of Nuclear Activity (CSAN, NC\%, N/F, AN/AF). Comparing the animals in terms of the Extracellular Matrix and Muscle Mass components (figure $3 \mathrm{~A}$ ), it is possible to notice that the CTL-SED animals are determined by the values of Muscular Mass, while the MSG-SED animals show direction governed by the increase in the Extracellular Matrix. Meanwhile, the exercise brought the animals CTL-WBV and MSG-WBV closer together, both in reducing muscle mass and in the extracellular matrix.
Meanwhile, the animals as a function of the Muscle Mass and Nuclear Activity components (figure $\mathbf{3} \mathbf{B}$ ) it is possible to notice that the CTL-WBV animals have greater Nuclear Activity and less Muscular Mass than the CTL-SED. Likewise, MSG-WBV animals have reduced Muscle Mass and Nuclear Activity. Even with different results of Nuclear Activity, it is possible to notice that the exercise promoted changes that bring the two WBV groups closer together. It is possible to observe this characteristic of approximation in the components Nuclear Activity and Extracellular Matrix (figure $3 \mathbf{C}$ ), where it is possible to notice that even with an increase in Nuclear Activity in CTL-WBV animals and a reduction in MSG-WBV animals, the approximation promoted by the reduction of Extracellular matrix in the MSG-WBV promotes overlapping of these animals with the CTL.

When evaluating the distribution of the Extracellular Matrix data (figure $3 \mathbf{A}^{\prime}$ ), there was an interaction between the factors $\left(\mathrm{p}<0.0001 ; \mathrm{F}_{(1,28)}=34,183\right)$. The MSG animals showed greater variation when compared to the CTL $\left(\mathrm{p}<0.0001 ; \mathrm{F}_{(1,28)}=165,931\right)$. Likewise, the exercise promoted a reduction in variations in WBV animals compared to SED $\left(\mathrm{p}<0.0001 ; \mathrm{F}_{(1,28)}=87,613\right)$. Conversely, when evaluating the distribution of Muscle Mass data (figure 3 B'), there was no interaction between the factors $\left(\mathrm{p}=0.92968 ; \mathrm{F}_{(1,28)}=0.008\right)$. However, it is possible to observe that MSG animals have lower averages than CTL $\left(\mathrm{p}<0.0001 ; \mathrm{F}_{(1,28)}=42.617\right)$. Likewise, WBV animals show reduced Muscle Mass when compared to SED ( $\mathrm{p}<$ 0.0001; $\left.F_{(1,28)}=44,798\right)$. Meanwhile, when assessing the distribution of Nuclear Activity data (figure 3 C'), there was an interaction between the factors $\left(\mathrm{p}=0.0001 ; \mathrm{F}_{(1,28)}\right.$ $=18.6430)$. Even though there was no difference between the CTL and MSG animals $\left(\mathrm{p}=0.185873 ; \mathrm{F}_{(1,28)}=1.8393\right)$ and the SED and WBV animals $\left(\mathrm{p}=0.168550 ; \mathrm{F}_{(1,28)}=\right.$ 1.9978), the interaction of the two factors promoted the opposite effect in the animals. While CTL-WBV showed an increase in Nuclear Activity compared to CTL-SED, animals MSG-WBV showed a reduction when compared to MSG-SED.

\section{DISCUSSION}

The obesity model used in the present study promoted negative changes in the structure of muscle tissue, increasing the conjunctival extracellular matrix and reducing muscle mass, and an increase in nuclear activity in MSG animals, demonstrating a harmful effect on tissue functionality. Nevertheless, when the MSG animals were submitted to WBV, these changes were reversed, with a reduction in the extracellular matrix and nuclear activity, suggesting muscle repair. Also, 


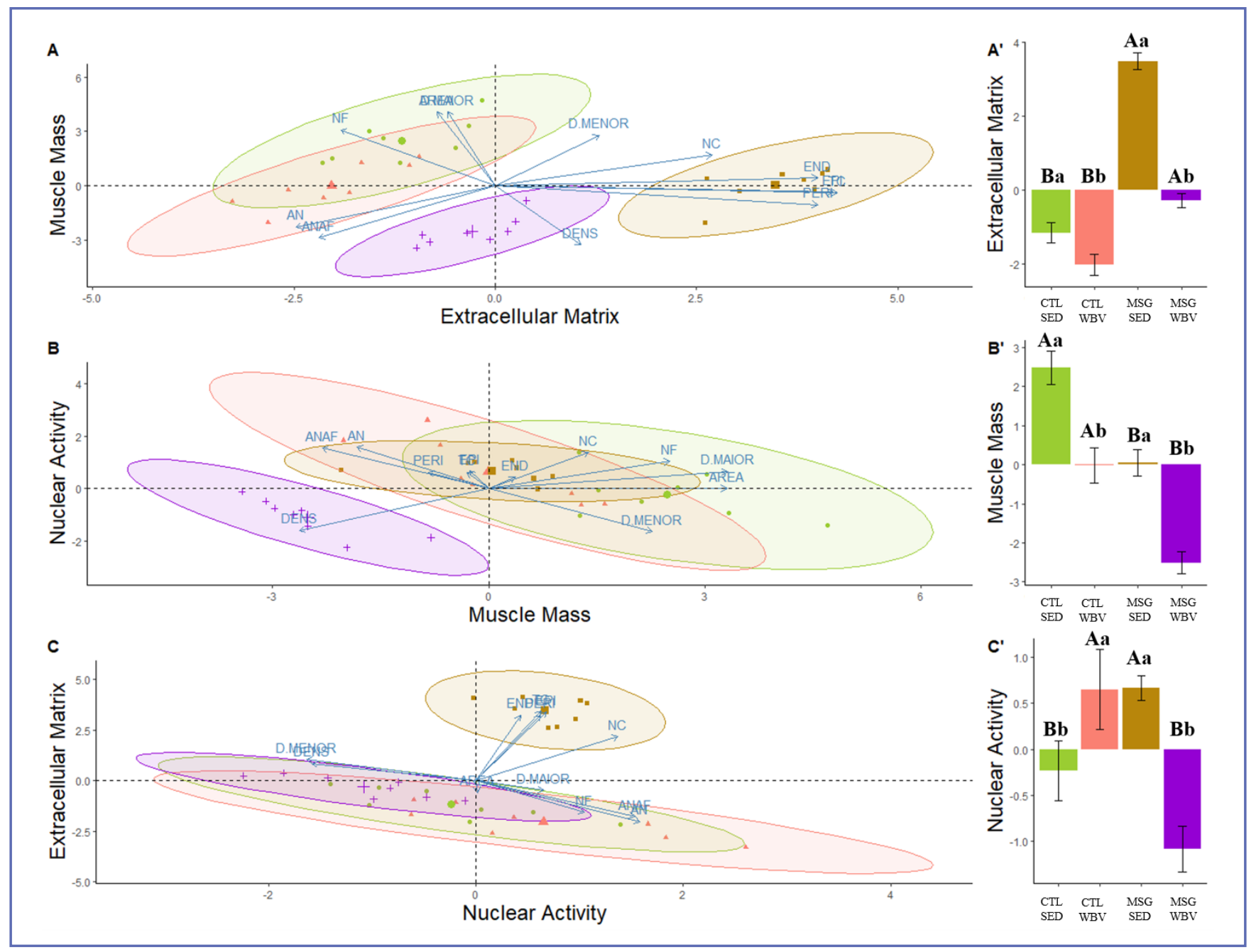

Figure 3. Principal Component Analysis (A-C) and Variation of data in each Component $\left(\mathbf{A}^{\prime}-\mathbf{C}^{\prime}\right)$.

CTL-SED: sedentary control group; CTL-WBV: trained control group; MSG-SED: sedentary obese group, MSG-WBV: trained obese group. Capital and different letters represent isolated differences from the MSG factor; lowercase and different letters represent isolated differences from the WBV. Extracellular Matrix (CON, EPI, PER, END), the second was called Muscle Mass (CSAF, LDF, SDF, DENS, AN/AF, N/F) and the third of Nuclear Activity (CSAN, NC\%, N/F, AN/AF), these three components exploit $84.47 \%$ of the data.

CTL animals increased nuclear activity, which may indicate the effect of exercise on protein synthesis.

The MSG-induced obesity model promoted an increase in the Lee index, as well as the deposition of retroperitoneal and perigonadal body fats, that corroborates with studies which, that have already demonstrated a significant increase in these parameters and, therefore, the efficiency of the model for the induction of obesity in rats $(14,15)$. The effectiveness of this experimental model is justified by the injuries that occur in several central structures in the paraventricular region, with the arcuate and ventromedial nuclei of the hypothalamus being the most affected sites, with $80 \%$ to $90 \%$ of the neurons responsible for controlling energy expenditure, food consumption and glycemic homeostasis (16). Thus, due to the damage to the hypothalamus caused by MSG, there is a decrease in the production and secretion of hormones such as $\mathrm{GH}$, resulting in a model of dwarfism due to low systemic growth derived from this low hormone (17).

When analyzing the histomorphometric parameters of the anterior tibial muscle, there were a reduction in the cross-sectional area, larger and smaller diameters of the muscle fiber in MSG animals, showing that the model was able to reproduce the expected hypotrophic characteristics. There is a direct relationship of inhibition of factors that 
repress muscle development, such as myostatin, in the presence of GH. Since these animals do not have the normal secretion of this hormone, there is an increase in the activity of myostatin, leading to less growth of muscle fibers during the development of the animal $(18,19)$.

The connective wraps are responsible for the structural maintenance of muscle fibers and for allowing the individually generated contraction force to act on the entire muscle and, finally, transmit it to the tendon and bone to generate movement. Also, the connective tissue serves as a support for nerves and blood vessels and which facilitates sliding for these structures. It is important to note that almost all pathological changes in the muscle are also associated with some degree of thickening of the extracellular matrix $(6,20)$. In this study, it was observed that obesity increased the percentage of all connective tissue variables in MSG-SED animals, possibly due to the reduction of protein synthesis and sedentary stimuli, which promotes muscle disuse, and consequently, atrophy $(5,7)$. Therefore, due to the obesity model causing muscle damage, there may be an imbalance in the proliferation of growth factors involved in muscle regeneration and mesenchymal growth, among them the fibroblast growth factor (FGF), which works by stimulating the synthesis of connective tissue (21). The connective tissue fills the area that would initially be occupied with muscle fiber, which justifies the increase in the connective tissue of the MSG-SED when compared to the CTL-SED. Besides, it was shown that the most significant increase in MSG-SED was concerning the perimysium, responsible for cell signaling $(20,22)$. In this sense, a structural change, such as an increase in fibrotic tissue, can negatively impact the arrival of muscle growth signaling factors.

The increase in the percentage of connective tissue observed in MSG-SED possibly occurred due to the high proliferation of fibroblasts and synthesis of type III collagen, which consequently led to the proliferation of type I collagen, which determines the tensile strength and rigidity of the tissue (23). This is also described in immobilization models (12) with an increase in intramuscular connective tissue in the anterior tibial, suggesting that there is a change in fibroblast metabolism and, consequently, an increase in collagen synthesis, which would be similar to what happened in the animals of the MSG-SED. Boaretto et al. (2020) (5), obtained similar results in the soleus muscle of Wistar rats, in which the sedentary obese group also obtained higher averages of muscle connective tissue. This highlights the role of a sedentary lifestyle and muscle disuse in the accumulation of connective tissue between muscle fibers. However, the WBV animals showed a reduction in the amount of connective tissue in the extracellular matrix, suggesting that the exercise used was able to alter the synthesis and remodeling of the muscle connective wraps. Polizello et al. (2011) (23), when assessing the distribution of collagen fibers in the wraps, observed that the exercise promoted a reduction in the proportion of type I collagen, which is mainly responsible for the rigidity and fibrotic characteristics of the tissue. In this sense, the remodeling promoted by the modality, in addition to reducing the amount of connective tissue, promotes increased flexibility and malleability. Also, WBV has shown promise in reducing obesogenic and inflammatory parameters in humans and improved insulin resistance. According to the authors, these results, which were also achieved in eight weeks, are largely driven by the reduction of body fat through the increase in fat oxidation promoted by the WBV (24).

The use of WBV in obese humans has beneficial effects on the modulation of body mass, promoting a reduction in body weight and fat deposits. Also, it promotes increased muscle mass and, consequently, increased muscle strength. The main mechanism of action of WBV on skeletal muscle involves the activation of neuromuscular spindles and $\alpha$ motor neurons (25). In this sense, the WBV promotes involuntary contractions through the tonic vibratory reflex. These stimuli promote, in addition to the induction of muscle protein synthesis and neural activation, modulation of cardiovascular activity, increased lipid mobilization, paracrine and endocrine activation $(7,26)$. The reduction in the cross-sectional area of CTL-WBV and MSG-WBV animals may be related to the muscle used (27). Although in the present study we presented a reduction in the size of the muscle fiber, other studies with the soleus muscle, an agonist of this movement, showed improvement in the cross-sectional area of animals trained with the same protocol (11).

Melo et al. (2019) (28) state that, unlike the safety limit parameters in the work environment, the use of therapeutic vibration, that is, by medical devices, is not yet standardized. According to the parameters of their revised protocols, and following the review by Zago et al. (2018) (26), in humans, an average of 15 minutes of daily exposure, $30-50 \mathrm{~Hz}$ and magnitude from 2.25 to 7.98 grams, amplitude around 2 millimeters $(\mathrm{mm})$ with a gradual increase in intensity during the intervention, can be safe, with positive effects for that population. On the other hand, data regarding the use of this therapeutic modality in obese rats is limited. In the work by Boaretto et al. (2020) (5), using the same protocol as the present study, the soleus muscle also showed a decrease in the cross-sectional area in the sedentary and obese groups submitted to the WBV. According to the authors, the result found was possibly due to the parameters used in the treatment protocol (alternate days per week, $60 \mathrm{~Hz}, 2 \mathrm{~mm}$ for 10 minutes), suggesting that higher frequencies are potentially harmful.

Despite the positive results found in the present study, the MSG model is a limitation, as it differs significantly from human obesity, which is mainly induced by excessive intake of caloric foods. In contrast, one of the strengths of the present study was the ability of the WBV exercise to promote 
improvement in the structural condition of the anterior tibial muscle, even in a model of such significant changes.

\section{CONCLUSIONS}

The results of the present study showed that treatment with WBV-exercise was able to promote significant improvements in the muscle structure of obese MSG animals.

\section{REFERENCE}

1. WHO. World Health Organization. Obesity and overweight 2020.

2. Tomlinson DJ, Erskine RM, Morse CI, Winwood K, Onambélé-Pearson $\mathrm{G}$. The impact of obesity on skeletal muscle strength and structure through adolescence to old age. Biogerontology 2016;17(3):467-83.

3. Souza MVC, Leite RD, Lino AD de S, et al. Resistance training improves body composition and increases matrix metalloproteinase 2 activity in biceps and gastrocnemius muscles of diet-induced obese rats. Clinics 2014;69(4):265-70.

4. Matsuda M, Shimomura I. Increased oxidative stress in obesity: Implications for metabolic syndrome, diabetes, hypertension, dyslipidemia, atherosclerosis, and cancer. Obes Res Clin Pract 2013;7(5):1-12.

5. Boaretto ML, de Andrade BZ, Maciel JIHN, et al. Effects of vibratory platform training on the histomorphometric parameters of the soleus muscle in obese Wistar rats. Sport Sci Health 2020;16:501-10;

6. Mann CJ, Perdiguero E, Kharraz Y, et al. Aberrant repair and fibrosis development in skeletal muscle. Skelet Muscle 2011;1(1):1-20.

7. de Andrade BZ, Zazula MF, Bittencourt Guimarães AT, et al. Whole-body vibration promotes lipid mobilization in hypothalamic obesity rat. Tissue Cell 2021;68:1-9.

8. Park S-Y, Son W-M, Kwon O-S. Effects of whole body vibration training on body composition, skeletal muscle strength, and cardiovascular health. J Exerc Rehabil 2015;11(6):289-95.

9. Kaneguchi A, Ozawa J, Kawamata S, Kurose T, Yamaoka K. Intermittent whole-body vibration attenuates a reduction in the number of the capillaries in unloaded rat skeletal muscle. BMC Musculoskelet Disord 2014;15:1-9.

10. Padulo J, Oliva F, Frizziero A, Maffulli N. Basic principles and recommendations in clinical and field science research: 2018 update. Muscles Ligaments Tendons J 2018;8(3):305-7.

11. Peretti AL, Kakihata CMM, Tavares AL de F, et al. Short-term effects of whole-body vibration on the soleus of ooforectomized rats: Histomorphometric analysis and oxidative stress in an animal model. Acta Histochem 2020;122(6):151598.

12. Kunz RI, Coradini JG, Silva LI, et al. Morfologia dos músculos sóleo e tibial anterior de ratos Wistar imobilizados e remobilizados em meio aquático. Conscientiae Saúde 2014;13(4):595-602.

13. R Core Team. R: A Language and Environment for Statistical Computing. R Foundation for Statistical Computing. Vienna 2020.

\section{ACKNOWLEDGMENTS}

The authors are grateful to UNIOESTE and UFPR for providing assistance and support.

\section{CONFLICT OF INTERESTS}

The authors declare that they have no conflict of interests.

14. Borck PC, Leite N de C, Valcanaia AC, et al. Swimming training reduces glucose-amplifying pathway and cholinergic responses in islets from lean- and MSG-obese rats. Clin Exp Pharmacol Physiol 2020;47(2):286-93.

15. Vitor-de-Lima SM, Medeiros LB, Benevides RDL, dos Santos CN, Lima da Silva NO, Guedes RCA. Monosodium glutamate and treadmill exercise: Anxiety-like behavior and spreading depression features in young adult rats. Nutr Neurosci 2019;22(6):435-43.

16. Lima CB, Soares G de SF, Vitor SM, Andrade-da-Costa BL da S, Castellano B, Guedes RCA. Spreading depression features and Iba1 immunoreactivity in the cerebral cortex of developing rats submitted to treadmill exercise after treatment with monosodium glutamate. Int J Dev Neurosci 2014;33(1):98-105.

17. Hirata AE, Andrade IS, Vaskevicius P, Dolnikoff MS. Monosodium glutamate (MSG)-obese rats develop glucose intolerance and insulin resistance to peripheral glucose uptake. Brazilian J Med Biol Res 1997;30(5):671-4.

18. Marcell TJ, Harman SM, Urban RJ, Metz DD, Rodgers BD, Blackman MR. Comparison of GH, IGF-I, and testosterone with mRNA of receptors and myostatin in skeletal muscle in older men. Am J Physiol Metab 2001;281(6):1159-64.

19. Grade CVC, Mantovani CS, Alvares LE. Myostatin gene promoter: structure, conservation and importance as a target for muscle modulation. J Anim Sci Biotechnol 2019;10(1):1-19.

20. Gillies AR, Lieber RL. Structure and function of the skeletal muscle extracellular matrix. Muscle Nerve 2011;44(3):318-31.

21. Du M, Yan X, Tong JF, Zhao J, Zhu MJ. Maternal obesity, inflammation, and fetal skeletal muscle development. In: Biology of Reproduction 2010:pp. 4-12.

22. Bayer ML, Bang L, Hoegberget-Kalisz M, et al. Musclestrain injury exudate favors acute tissue healing and prolonged connective tissue formation in humans. FASEB J 2019;33:10369-82.

23. Polizello JC, Carvalho LC, Freitas FC, Padula N, Martinez EZ, Mattiello-Sverzut AC. Morphological effects of resumption of loading after immobilization of skeletal muscles in lengthened position in female rats. Rev Bras Fisioter 2011;15(1):73-9.

24. Bellia A, Sallì M, Lombardo M, et al. Effects of whole body vibration plus diet on insulin-resistance in middle-aged obese subjects. Int J Sports Med 2014;35(6):511-6. 
25. Harwood B, Scherer J, Brown RE, Cornett KMD, Kenno KA, Jakobi JM. Neuromuscular responses of the plantar flexors to whole-body vibration. Scand J Med Sci Sport 2017;27(12):1569-75.

26. Zago M, Capodaglio P, Ferrario C, Tarabini M, Galli M. Wholebody vibration training in obese subjects: A systematic review. PLoS One 2018;13(9):1-2.
27. Duchateau J, Baudry S. The neural control of coactivation during fatiguing contractions revisited. J Electromyogr Kinesiol 2014;24(6):780-8.

28. Tenório de Melo FA, Ferreira de Melo G, Leão de Albuquerque Neto S. Protocolos de treinamento de vibração de corpo inteiro em obesos: uma revisão sistemática. Rev Bras de Medicina do Esporte 2019;25(6):527-33 